\title{
Fano Resonance-Based Blood Plasma Monitoring and Sensing using Plasmonic Nanomatryoshka
}

\author{
Pankaj Pathania ${ }^{1,2} \cdot$ Manmohan Singh Shishodia $^{1}$ [D
}

Received: 18 August 2020 / Accepted: 20 November 2020 / Published online: 10 June 2021

○) Springer Science+Business Media, LLC, part of Springer Nature 2021

\begin{abstract}
The fast label-free detection of specific antibodies and their concentration in blood plasma is useful for many applications, e.g., in Covid-19 patients. The change in biophysical properties like the refractive index of blood plasma due to the production of antibodies during infection may be very helpful in estimating the level and intensity of infection and subsequent treatment based on blood plasma therapy. In this article, Fano resonance-based refractive index sensor using plasmonic nanomatryoshka is proposed for blood plasma sensing. The interaction between hybridized modes (bright and dark modes) in optimized nanomatryoshka leads to Fano resonance, which by virtue of steeper dispersion can confine the light more efficiently compared with Lorentzian resonance. We propose the excitation of Fano resonances in sub 100-nm size nanomatryoshka based on newly emerging plasmonic materials $\mathrm{ZrN}$ and $\mathrm{HfN}$, and one of the most widely used conventional plasmonic material, Au. Fano resonance-based plasmonic sensors leads to sensitivity $=188.5 \mathrm{~nm} / \mathrm{RIU}, 242.5 \mathrm{~nm} / \mathrm{RIU}$, and $244.9 \mathrm{~nm} / \mathrm{RIU}$ for $\mathrm{Au}, \mathrm{ZrN}$, and HfN, respectively. The corresponding figure of merit (nm/RIU) is $\sim 3.5 \times 10^{3}, 3.1 \times 10^{3}$, and $2.8 \times 10^{3}$ for Au, $\mathrm{ZrN}$, and $\mathrm{HfN}$, respectively. Present theoretical analysis shows that refractive index sensors with high sensitivity and figure of merit are feasible using Fano modes of plasmonic nanomatryoshka.
\end{abstract}

Keywords Plasmonics $\cdot$ Sensor $\cdot$ Fano resonance $\cdot$ Mie-theory $\cdot$ Transition metal nitrides

\section{Introduction}

In the backdrop of the fast growing world population, the need for developing efficient healthcare systems, particularly for medical testing and diagnostics, is at its high demand [1, 2]. The recent worldwide spread of COVID-19 pandemic has emphasized on the vitality of fast testing and diagnostic techniques. This is the motivation that encourages the researchers to explore the possibility and scope of developing efficient, long-lasting, economical, and practically feasible solutions to the existing as well as future medical challenges posing threat to the existence of humanity. The photonic systems provide solution for the development of label free fast detection/sensing system in which various types of resonances, e.g., Fabry-Perot resonators, Bragg

Manmohan Singh Shishodia

manmohan@gbu.ac.in

1 Department of Applied Physics, Gautam Buddha University, 201312 Greater Noida, India

2 Galogotias College of Engineering and Technology, 201312 Greater Noida, India resonators, reflection filters, antireflection filters, and transmission gratings, have played the key role [3]. Beyond classical diffraction limit, sub wavelength metallic structures show nanoscale optical confinement by virtue of excitation of surface plasmons having unique properties [4]. Such structures will provide a better platform for switching, spasing, molecular energy transfer, energy harvesting, bacteria detection, cancer therapy, etc. [5-16]. Plasmonic systems are emerging as the backbone of medical diagnostics, e.g., large efforts of physicists, chemists, biologists, and material scientists, are focused on the development of an ultrasensitive plasmonic sensor for bio-molecular sensing. The origin of asymmetric resonant (Fano resonance) interaction [17] in the complex geometry like concentric or non-concentric nanoshell [18-20], rings [21], septamer [22], trimers [23], and disks-ring [24] provides hybrid plasmonic systems characterized by rich plasmonic spectra. This not only open the possibility for new devices based on Fano modes but may also improve the performance of existing plasmonic device $[25,26]$. Fano resonance usually involves the interference of two modes, the one with spectrally broad background and the other with narrow resonanceand characterized by strong 
light confinement [27]. Fano resonance shows steeper dispersion and strong dependence on size, shape, and surrounding medium, compared with Lorentzian resonance [27]. In the last decade, asymmetric Fano resonances in plasmonic nanostructures have been a subject of intensive reaserch. For example, N. Halas and S. Link [28] at Rice University reported the first "plasmonic Fano switch for color display" based on tiny gold nanoparticles and liquid crystals. It is now well established that the Fano resonances originate from near-field coupling of "bright" and "dark" plasmon modes of individual nanoparticle within the cluster [28]. The excitations of Fano resonances in optimized structure provide a platform for detection [29], surface-enhanced Raman spectroscopy [30], nanolithography, microscopy, sub-diffraction imaging [31], ultra-small size laser [31], interferometric phase detection, ultrasensitive spectroscopy, identification of molecular monolayer [32], X-Ray tomography, structural analysis, and precision metrology [33]. Irrespective of geometry, polarization insensitive sub $100 \mathrm{~nm}$ multilayered core-shell structure called nanomatryoshka (core/shell/shell) shows the inherent capability of spectral tuning and unique characteristics [16]. In optimized nanomatryoshka the interaction and coupling of low and high energy hybridized modes lead to the excitation of Fano modes [20, 21]. In addition to the choice of an appropriate geometry of plasmonic nanostructure, the proper selection of plasmonic material is also very crucial for designing fast and efficient optoelectronic systems. Traditionally, the coinage metals like gold, silver, copper, and aluminum have been the primary choice of plasmonic materials in the plasmonic systems and devices. The real-time fabrication issue and incompatibility with standard fabrication [34], with inherent loss due to large real part in noble metals, are the key motivations for using newly emerging plasmonic materials like refractory transition metal nitrides $(\mathrm{ZrN}, \mathrm{HfN}$, etc.) for existing plasmonic-based devices [35-40]. In our previous work, it was examined that the refractory transition metal nitrides (RTMNs) like ZrN and HfN show plasmonic properties similar to the conventional plasmonic material Au [7, 8]. Hence, refractory transition metal nitrides (i.e., $\mathrm{ZrN}$, TiN, and $\mathrm{HfN})$ and the ternary alloy $\left(\mathrm{Ti}_{\mathrm{x}} \mathrm{Zr}_{1-\mathrm{x}} \mathrm{N}\right)$ exhibit attractive plasmonic properties which play a crucial role in designing a better platform for refractive index sensing/detection. The unique biophysical signature (like refractive index and morphology) of bio-molecules (e.g., cells, bacteria, blood plasma, proteins) pave the way for label-free detection and sensing [41-43]. In a normal human being, the blood consists of about $55 \%$ plasma ( $90 \%$ water and $10 \%$ proteins) and $45 \%$ cells (99\% erythrocytes, $1 \%$ leukocytes, and thrombocytes). Since, blood plasma contains proteins (antibodies) and other ingredients, due to slight variation in the blood plasma concentration cause variation in the $\mathrm{pH}$ value of blood, which in turn leads to serious health deterioration.
The detection of specific antibodies and the concentration of blood plasma in Covid-19 patients are key for monitoring their health and extremely useful in blood plasma therapy. It is therefore very crucial to develop a label free sensing system to monitor the concentration of blood plasma. Microfluidic techniques for estimating the concentration of hydrogen sulphide [44], glucose, and cholesterol in blood plasma have already been reported [45]. For example, silver-based luminescent probe has been designed for enzymatic sensing/detection of glucose level in blood plasma [46]. Further, the cancer bio-marker changes the thermal behavior of most abundant proteins in blood plasma and its detection in blood plasma and urine is normally done by techniques such as differential scanning calorimeter and mass spectrometry [47]. The excitation of SPR in optical fiber leads to the detection of fibrinogen traces in the blood plasma of Alzheimer's patients with a detection limit of $20 \mathrm{ng} / \mathrm{ml}$ [48]. In the backdrop of above discussion, it is therefore imperative to develop improved methods for detecting minute changes in the blood plasma concentration for early diagnosis of the diseases.

The present work analyzes the feasibility of efficient refractive index sensing of blood plasma concentration using Fano modes of transition metal nitride-based nanomatryoshka. The crucial role of Fano mode's lineshape requires effective and efficient theoretical tools. The present work intends to accomplish the following objectives: (I) to study the origin of asymmetric Fano line shape in subwavelength sized nanomatryoshka, (II) to investigate the unique features of Fano resonances and their comparative study with symmetric localized modes, (III) to assess the sensitivity and figure of merit of Fano resonances for different sensing environments, and (IV) quantitative analysis of sensing properties as suitable for blood plasma sensing.

\section{Theoretical Model}

The nanomatryoshka-based plasmonic system considered for present investigations consists of three concentric layers, namely, the metallic core having permittivity $\varepsilon_{1}$ and radius $R_{1}$, dielectric layer having permittivity $\varepsilon_{2}$ and radius $R_{2}$, and metallic shell having permittivity $\varepsilon_{3}$ and radius $R_{3}$ as shown in Fig. 1. The nanoparticle is ingrained in the medium of dielectric constant, $\varepsilon_{m}=n^{2}$, where $n$ is the refractive index of the ingrained medium. The numerous analytical (quasi-static approach, Mie scattering theory, transfer matrix approach, etc.), and numerical (FEM, FDTD, etc.) methods are available for studying and simulating optical properties of nanomatryoshaka. In the present work, the well-established Mie theory has been used to treat plasmonic nanomatryoshka [49]. Mie theory consider that the uniform field incident on nanomatryoshka produces scattered and internal fields. These fields may be expressed as an infinite series of vector 


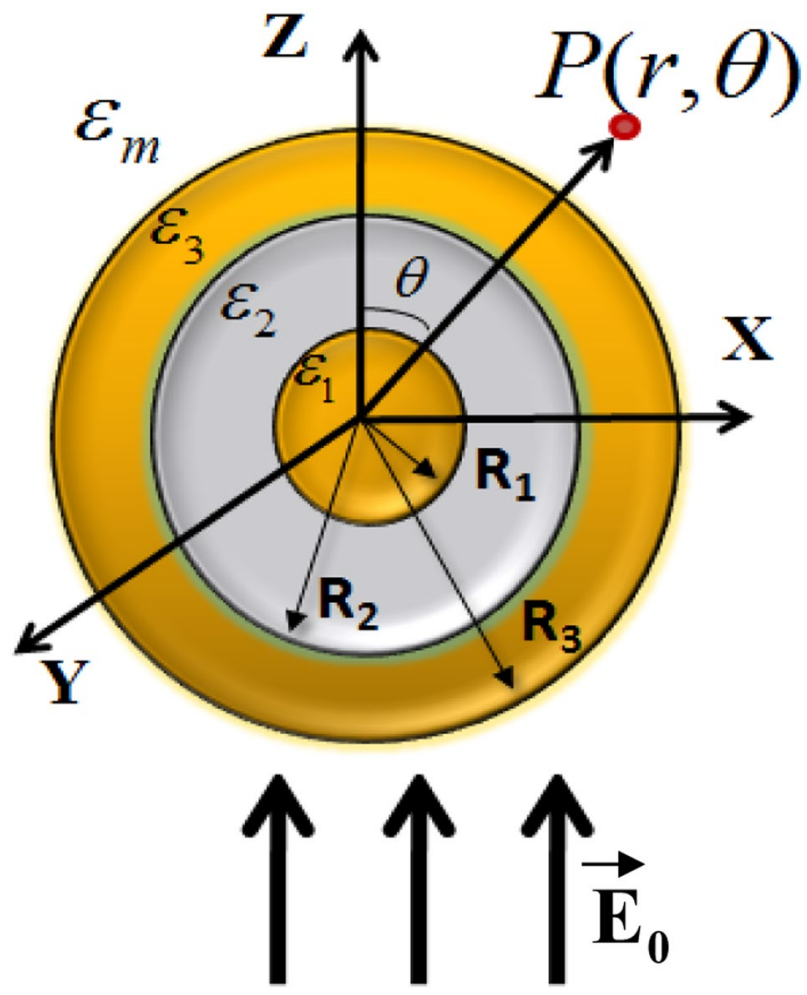

Fig. 1 The schematic diagram of plasmonic nanomatryoshka particle. The nanomatryoshka consists of metallic core having permittivity $\varepsilon_{1}$ and radius $R_{1}$, dielectric layer having permittivity $\varepsilon_{2}$ and radius $R_{2}$, and metallic shell having permittivity $\varepsilon_{3}$ and radius $R_{3}$. Nanomatryoshka particle is ingrained in the medium of dielectric constant $\varepsilon_{m}$. It is assumed that the particle is placed in the uniform electric field $\vec{E}_{0}$

spherical harmonics. This is to be emphasized that the Mie analysis is based on the full wave solutions of electromagnetic Maxwell equation formulated for the system under consideration. The solutions are commonly expressed in the form of Mie coefficients $\left(a_{l}\right.$ and $\left.b_{l}\right)$ as defined below [50, 51], Eq. 1

$\begin{aligned} a_{l} & =\frac{\psi_{l}\left(x_{3}\right) H_{l}^{a}\left(f_{3} x_{3}\right)-f_{3} D_{l}^{(1)}\left(x_{3}\right)}{\xi_{1}\left(x_{3}\right) H_{l}^{a}\left(f_{3} x_{3}\right)-f_{3} D_{l}^{(3)}\left(x_{3}\right)} \\ b_{l} & =\frac{\psi_{l}\left(x_{3}\right) f_{3} H_{l}^{b}\left(f_{3} x_{3}\right)-D_{l}^{(1)}\left(x_{3}\right)}{\xi_{l}\left(x_{3}\right) f_{3} H_{l}^{b}\left(f_{3} x_{3}\right)-D_{l}^{(3)}\left(x_{3}\right)}\end{aligned}$

Here, for four layer system, and size parameter $x_{3}=k R_{3}$ where $k$ is the wavenumber, $f_{3}=\varepsilon_{3} / \varepsilon_{m}$ is the relative refractive index of outer shell medium with respect to the ingrained medium, Ricatti-Bessel functions $\psi_{l}(z)=z j_{l}(z) ; \chi_{l}(z)=-z y_{l}(z), \xi_{l}(z)=z h_{l}^{1}(z)$ are written in the form of spherical Bessel functions $j_{l}(z), y_{l}(z)$, and $h_{l}^{1}(z)$. The logarithmic derivative of Ricatti-Bessel function like $D_{l}^{(1)}, D_{l}^{(2)}$ and $D_{l}^{(3)}$ are written as

$$
D_{l}^{(1)}=\frac{\psi_{l}^{\prime}(z)}{\psi_{l}(z)} ; D_{l}^{(2)}=\frac{\chi_{l}^{\prime}(z)}{\chi_{l}(z)} ; D_{l}^{(3)}=\frac{\xi_{l}^{\prime}(z)}{\xi_{l}(z)}, \text { and } H_{l}^{a}\left(f_{3} x_{3}\right), H_{l}^{b}\left(f_{3} x_{3}\right)
$$

are calculated as in Eq. 2

$H_{l}^{a}\left(f_{3} x_{3}\right)=\frac{\left[\psi_{l}\left(f_{3} x_{3}\right) / \chi_{l}\left(f_{3} x_{3}\right)\right] D_{l}^{(1)}\left(f_{3} x_{3}\right)}{\left[\psi_{l}\left(f_{3} x_{3}\right) / \chi_{l}\left(f_{3} x_{3}\right)\right]-A_{l}^{(3)}}-\frac{A_{l}^{(3)} D_{l}^{(2)}\left(f_{3} x_{3}\right)}{\left[\psi_{l}\left(f_{3} x_{3}\right) / \chi_{l}\left(f_{3} x_{3}\right)\right]-A_{l}^{(3)}}$
$H_{l}^{b}\left(m_{3} x_{3}\right)=\frac{\left[\Psi_{l}\left(f_{3} x_{3}\right) / \chi_{l}\left(f_{3} x_{3}\right)\right] D_{l}^{(1)}\left(f_{3} x_{3}\right)}{\left[\psi_{l}\left(f_{3} x_{3}\right) / \chi_{l}\left(f_{3} x_{3}\right)\right]-B_{l}^{(3)}}-\frac{B_{l}^{3} D_{l}^{(2)}\left(f_{3} x_{3}\right)}{\left[\psi_{l}\left(f_{3} x_{3}\right) / \chi_{l}\left(f_{3} x_{3}\right)\right]-B_{l}^{(3)}}$

the corresponding terms $A_{l}^{(3)}, B_{l}^{(3)}$ can be written as in Eq. 3

$A_{l}^{(3)}=\frac{\Psi_{l}\left(f_{3} x_{2}\right)}{\chi_{l}\left(f_{3} x_{2}\right)} \frac{f_{3} H_{l}^{a}\left(f_{2} x_{2}\right)-f_{2} D_{l}^{(1)}\left(f_{3} x_{2}\right)}{f_{3} H_{l}^{a}\left(f_{2} x_{2}\right)-f_{2} D_{l}^{(2)}\left(f_{3} x_{2}\right)}$
$B_{l}^{(3)}=\frac{\Psi_{l}\left(f_{3} x_{2}\right)}{\chi_{l}\left(f_{3} x_{2}\right)} \frac{f_{2} H_{l}^{b}\left(f_{2} x_{2}\right)-f_{2} D_{l}^{(1)}\left(f_{3} x_{2}\right)}{f_{2} H_{l}^{b}\left(f_{2} x_{2}\right)-f_{2} D_{l}^{(2)}\left(f_{3} x_{2}\right)}$

where Eq. 4

$H_{l}^{a}\left(f_{2} x_{2}\right)=\frac{\left[\psi_{l}\left(f_{2} x_{2}\right) / \chi_{l}\left(f_{2} x_{2}\right)\right] D_{l}^{(1)}\left(f_{2} x_{2}\right)}{\left[\psi_{l}\left(f_{2} x_{2}\right) / \chi_{l}\left(f_{2} x_{2}\right)\right]-A_{l}^{(2)}}-\frac{A_{l}^{(2)} D_{l}^{(2)}\left(f_{2} x_{2}\right)}{\left[\psi_{l}\left(f_{2} x_{2}\right) / \chi_{l}\left(f_{2} x_{2}\right)\right]-A_{l}^{(2)}}$
$H_{l}^{b}\left(m_{2} x_{2}\right)=\frac{\left[\psi_{l}\left(f_{2} x_{2}\right) / \chi_{l}\left(f_{2} x_{2}\right)\right] D_{l}^{(1)}\left(f_{2} x_{2}\right)}{\left[\psi_{l}\left(f_{2} x_{2}\right) / \chi_{l}\left(f_{2} x_{2}\right)\right]-B_{l}^{(2)}}-\frac{B_{l}^{3} D_{l}^{(2)}\left(f_{2} x_{2}\right)}{\left[\Psi_{l}\left(f_{3} x_{3}\right) / \chi_{l}\left(f_{2} x_{2}\right)\right]-B_{l}^{(2)}}$

where the coefficients $A_{l}^{(2)}, B_{l}^{(2)}$ are written as in Eq. 5

$\begin{aligned} A_{l}^{(2)} & =\frac{\Psi_{l}\left(f_{2} x_{1}\right) f_{2} H_{l}^{a}\left(f_{1} x_{1}\right)-f_{1} D_{l}^{(1)}\left(f_{2} x_{1}\right)}{\chi_{l}\left(f_{3} x_{2}\right) f_{3} H_{l}^{a}\left(f_{1} x_{1}\right)-f_{1} D_{1}^{(2)}\left(f_{2} x_{1}\right)} \\ B_{l}^{(2)} & =\frac{\Psi_{l}\left(f_{3} x_{2}\right) f_{2} H_{l}^{b}\left(f_{1} x_{1}\right)-f_{1} D_{l}^{(1)}\left(f_{2} x_{1}\right)}{\chi_{l}\left(f_{3} x_{2}\right) f_{2} H_{l}^{b}\left(f_{1} x_{1}\right)-f_{1} D_{l}^{(2)}\left(f_{2} x_{1}\right)}\end{aligned}$

where in Eq. 6

$A_{l}^{(1)}=0, H_{l}^{a}\left(f_{1} x_{1}\right)=D_{l}^{1}\left(f_{1} x_{1}\right)$

$B_{l}^{(1)}=0, H_{l}^{b}\left(f_{1} x_{1}\right)=D_{l}^{1}\left(f_{1} x_{1}\right)$

Here, $x_{1}=k R_{1}, x_{2}=k R_{2}, x_{3}=k R_{3}$ are the size parameters and $k$ is the wave vector and, $f_{1}, f_{2}$ and $f_{3}$ are the refractive indices of the core, sandwich layer, and shell relative to the surrounding medium, respectively. Moreover, the extinction efficiencies $\left(Q_{\text {ext }}\right)$, scattering $\left(Q_{\text {sca }}\right)$, and absorption $\left(Q_{\mathrm{abs}}\right)$ efficiencies are written as [49] in Eq. 7

$Q_{\text {ext }}=\frac{2}{\left(k R_{3}\right)^{2}} \sum_{l=1}^{\infty}[2 l+1] \operatorname{Re}\left(a_{l}+b_{1}\right)$

$Q_{s c a}=\frac{2}{\left(k R_{3}\right)^{2}} \sum_{l=1}^{\infty}[2 l+1]\left(\left|a_{1}\right|^{2}+\left|b_{l}\right|^{2}\right)$

$Q_{a b s}=Q_{e x t}-Q_{s c a}$ 
where $k$ is the wavenumber of medium and $R_{3}$ is the radius of nanomatryoshka.

\section{Results and Discussion}

In order to validate the present approach and the code that we developed, the results reported in Wu et al. [50] are reproduced for Au-based nanomatryoshka (NMS) and nanoshell $(\mathrm{NSH})$ with outer radius of $50 \mathrm{~nm}$ as shown in Fig. 2a. The validation for three different core sizes $(15,21,25 \mathrm{~nm})$ is shown. The optical constants of refractory transition metal nitrides $\mathrm{ZrN}$ and $\mathrm{HfN}$ are adopted from Kumar et al. [39] and that of Au are adopted from Shishodia and Juneja [13]. The spectral variation of scattering efficiency for $\mathrm{ZrN}$-based nanomatryoshka $\left(R_{1}=30 \mathrm{~nm}, R_{2}=50 \mathrm{~nm}\right.$, and $\left.R_{3}=70 \mathrm{~nm}\right)$ is presented in Fig. 2b. For the sake of comparison, the corresponding overlay plots for spherical $(R=70 \mathrm{~nm})$ and nanoshell $\left(R_{1}=50 \mathrm{~nm}, R_{2}=70 \mathrm{~nm}\right)$ geometries are also presented. The scattering spectra show the possibility of the appearance of Fano resonance in nanomatryoshka. This is possible by virtue of indirect coupling between non-radiative (dark) and radiative (bright) modes of plasmonic nanostructure [52-54]. Figure $2 \mathrm{~b}$ clearly shows that, Fano modes appear in nanomatryoshka but not in sphere and nanoshell. Evidently, Fano dip and peak appears at, $\lambda=635 \mathrm{~nm}$ and $\lambda=743 \mathrm{~nm}$. In order to confirm that the resonance appeared in nanomatryoshka is Fano resonance, the calculated data is fitted into the following standard lineshape expression of Fano resonance [17],

$f(\lambda)=\frac{\left(F \times w+\lambda-\lambda_{R}\right)^{2}}{\left(\lambda-\lambda_{R}\right)^{2}+w^{2}}$

In Eq. 8, $F$ is called Fano parameter (degree of asymmetry), $\lambda_{R}$ is the position of resonance, and $w$ is the width
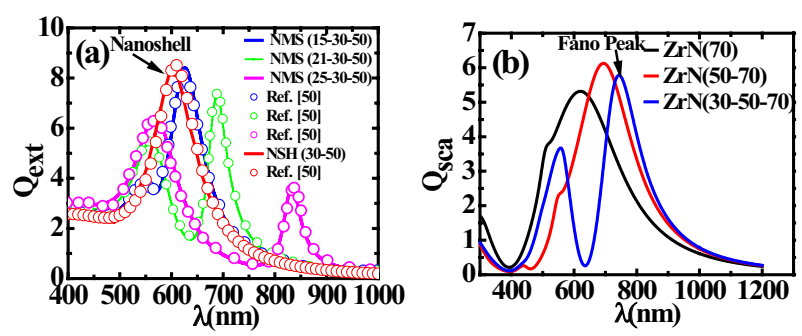

Fig. 2 a Validation of Mie theory-based extinction efficiency $\left(Q_{\text {ext }}\right)$ for Au-based nanomatryoshka (NMS) and nanoshell (NSH) with specifications same as in Ref. [50]. b The calculated spectral variation of scattering efficiency for $\mathrm{ZrN}$-based nanosphere $(70 \mathrm{~nm})$, nanoshell $(50-70 \mathrm{~nm})$, and nanomatyroshka $(30-50-70 \mathrm{~nm})$ particle ingrained in the medium of refractive index 1.333

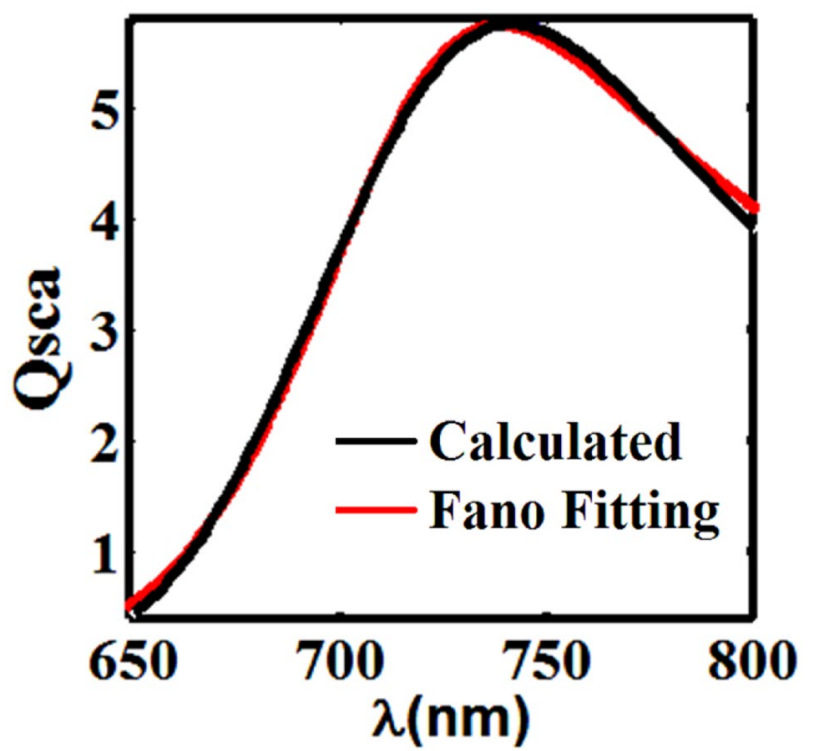

Fig. 3 The calculated spectral variation of scattering efficiency of ZrN-based nanomatyroshka (30-50-70 nm) and corresponding fitting of calculated data into Fano expression. The excellent fitting of calculated data into the standard Fano expression confirm the Fano nature of resonance

of Fano resonance. The calculated spectral variation of scattering efficiency and the corresponding fitting into Eq. 8 is presented in Fig. 3 for $\mathrm{ZrN}$-based nanomatryoshka ingrained in the medium of refractive index 1.333 . The fairly good fitting of calculated data into Fano expression confirms the Fano nature of resonance in nanomatryoshka. The fitting parameters are summarized in Table 1 . The calculated spectral variation of scattering efficiency $\left(Q_{\text {sca }}\right)$ for nanomatryoshka consisting of $\mathrm{Au}, \mathrm{ZrN}$, and $\mathrm{HfN}$ shell is shown in Fig. 4a. Unless mentioned otherwise, the sandwich dielectric medium is assumed to be lossless silica with wavelengthindependent dielectric constant, $\varepsilon_{2}=2.04$. The size parameters considered for this analysis are $R_{1}=30 \mathrm{~nm}, R_{2}=50 \mathrm{~nm}$, and $R_{3}=70 \mathrm{~nm}$. This is evident that the scattering efficiency (normalized scattering cross-section) spectra show resonant behavior by virtue of excitation of localized surface plasmon modes, whose interaction results in the characteristic Fano resonance. For the nanomatryoshka under consideration, Fano peak appears at $\lambda=737 \mathrm{~nm}, 741 \mathrm{~nm}, 703 \mathrm{~nm}$, and Fano

Table 1 The Fano fitting parameter of nanomatryoshka with $R_{1}=30 \mathrm{~nm}, R_{2}=50 \mathrm{~nm}$, and $R_{3}=70 \mathrm{~nm}$

\begin{tabular}{llll}
\hline Fitting Parameters & $\mathrm{Au}$ & $\mathrm{ZrN}$ & HfN \\
\hline$F$ & 2.24 & 2.17 & 2.12 \\
$\lambda_{R}(\mathrm{~nm})$ & 738 & 743 & 705 \\
$W(\mathrm{~nm})$ & 45.16 & 57.45 & 61.23 \\
\hline
\end{tabular}



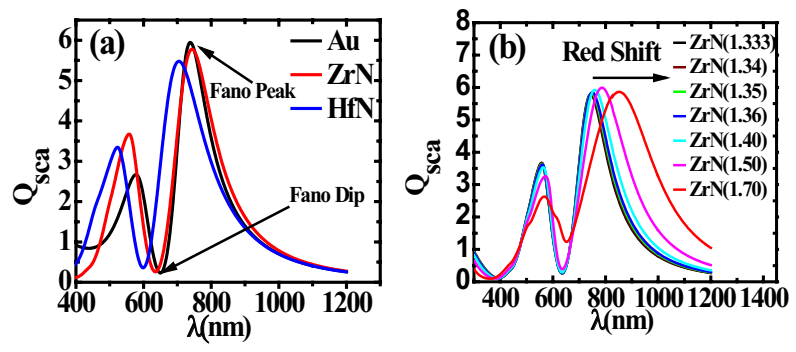

Fig. 4 a The calculated spectral variation of scattering efficiencies of $\mathrm{Au}, \mathrm{ZrN}$, and $\mathrm{HfN}$-based nanomatryoshka with $R_{1}=30 \mathrm{~nm}$, $R_{2}=50 \mathrm{~nm}$, and $R_{3}=70 \mathrm{~nm}$. b The spectral variation of scattering efficiency of $\mathrm{ZrN}$-based nanomatryoshka $(30,50,70)$ ingrained in the surrounding medium of slightly different refractive indices. The red-shift of Fano resonance peak with increasing refractive index of ingrained medium is clearly evident

dip appears at $\lambda=650 \mathrm{~nm}, 635 \mathrm{~nm}, 599 \mathrm{~nm}$ for Au-, $\mathrm{ZrN}-$, and HfN-based nanomatryoshka, respectively. It is evident that the Fano peak scattering efficiencies of $\mathrm{Au}, \mathrm{ZrN}$, and $\mathrm{HfN}$ are comparable having magnitude 5.9, 5.7, and 5.4, respectively. Moreover, FWHM (nm) values are $45.57 \mathrm{~nm}$, $57.16 \mathrm{~nm}$, and $61.23 \mathrm{~nm}$, respectively. Figure $4 \mathrm{~b}$ clearly shows the red-shift of Fano resonance peak as the refractive index of the surrounding medium around $\mathrm{ZrN}$-based nanomatryoshka is varied slightly. It is evident that the resonant Fano peak wavelength is extremely sensitive even to a slight change in the refractive index of the surrounding medium. As it is well known that the healthy blood plasma comprises of water and crucial proteins with refractive index, 1.32459 [55], the small change in the concentration of blood plasma leads to a change in the refractive index. Next, let us consider a practical scenario, where the sensing medium is the defected blood containing different blood plasma concentrations. The refractive index change resulting from the change in plasma concentration, e.g., resulting from contaminations and diseases may be written as [55],

$n=1.32459+0.000194 C_{p}$

In Eq. 9, $C_{p}$ denotes the concentration of defected plasma in the blood and it is normally expressed in the units of grams per liter. In the present calculations, $C_{P}$ is varied from 0 to $50 \mathrm{~g} / \mathrm{l}$ in the steps of $10 \mathrm{~g} / \mathrm{l}$. The refractive index values of blood for different concentrations of contaminants in blood plasma are tabulated in Table 2. The calculated spectral variations of scattering efficiency $\left(Q_{\text {sca }}\right)$ for nanomatryoshka consisting of $\mathrm{Au}, \mathrm{ZrN}$, and $\mathrm{HfN}$ are shown in Fig. 5a-c, respectively. The results are shown for six different plasma concentrations in blood. It is evident that the Fano resonance appears in the scattering spectra of Au-, $\mathrm{ZrN}-$, and HfN-based nanomatryoshka. This is clearly
Table 2 The optimized refractive index of defected concentration of blood plasma [55]

\begin{tabular}{lll}
\hline Sr. no & Concentration $(\mathrm{g} / l)$ & Refractive index $(n)$ \\
\hline 1 & 0 & 1.324590000 \\
2 & 10 & 1.339692811 \\
3 & 20 & 1.359112811 \\
4 & 30 & 1.378592811 \\
5 & 40 & 1.397952811 \\
6 & 50 & 1.417372811 \\
\hline
\end{tabular}

evident that the peak position of Fano resonance shifts on varying the plasma concentration. Moreover, Fano resonance wavelength varies almost linearly with the concentration of blood plasma as is evident from Fig. 5d. A significant shift in Fano resonance wavelength is observed with minuscule change of blood plasma concentration. Interestingly, the Fano dip wavelength remains almost unaltered with the change in blood plasma concentration. It is interesting to note that the scattering efficiency at resonance dip wavelength in all cases is very close to zero. This corresponds to the high optical absorption in this spectral region. This feature is desired for designing efficient light absorbing nanostructures. It is evident that the scattering peak wavelength is also a highly sensitive function of the refractive index of the surrounding/sensing medium. The fractional change in the refractive index leads to the drastic reduction in scattering efficiency, and the shift in resonant wavelength makes the basis of Fano resonance-based biosensing. The calculated Fano peak wavelength positions for six different plasma concentrations in Au-, $\mathrm{ZrN}-$, and HfN-based nanomatryoshka $(30,50,70)$ are summarized in Table 3 . The sensitivity $(S)$ of refractive index sensor is defined as the rate of change of resonant peak wavelength $(\mathrm{nm})$ with the refractive index of sensing medium. Mathematically, the sensitivity (nm/RIU) of the sensor can be written as [14]

$S=\frac{d \lambda_{R}}{d n}$

The dependence of Fano peak wavelength on the concentration of defected blood plasma and hence the refractive index can be fitted into a linear equation of the following form,

$\lambda_{R}(n m)=\lambda_{0}+S \times n$

In Eq. $11, \lambda_{0}$ and $S$ are the fitting parameters. The dependence of resonant wavelength $\left(\lambda_{R}\right)$ with change in refractive index of sensing blood plasma medium and corresponding sensitivity (S) for (a). Au, (b). $\mathrm{ZrN}$, and (c). HfN based nanomatryoshka are shown in Fig. 6. The analysis 
Fig. 5 The calculated spectral variation of scattering efficiency $\left(Q_{\text {sca }}\right)$ spectra of a $\mathrm{Au}, \mathbf{b} \mathrm{ZrN}$, and $\mathbf{c}$ HfN-based nanomatryoshka with $R_{1}=30 \mathrm{~nm}$, $R_{2}=50 \mathrm{~nm}$, and $R_{3}=70 \mathrm{~nm}$. The variation of resonant peak wavelength with concentration of contaminated blood plasma is shown in $\mathbf{d}$
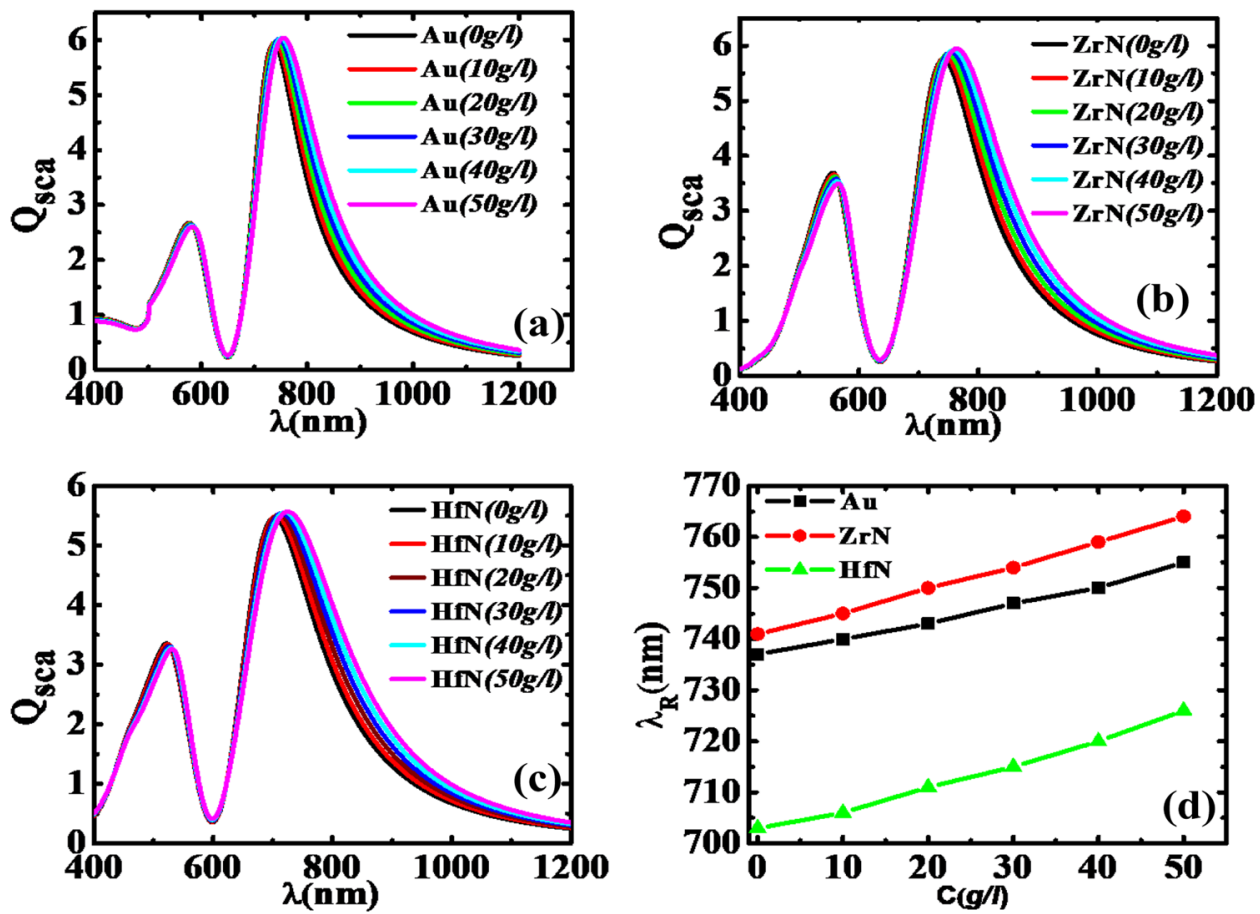

of data shown in Fig. 6 shows that the sensitivity values corresponding to the Fano resonances in $\mathrm{Au}-, \mathrm{ZrN}-$, and HfN-based nanomatryoshka are $188.5 \mathrm{~nm} / \mathrm{RIU}, 242 \mathrm{~nm} /$ RIU, and $244.9 \mathrm{~nm} / \mathrm{RIU}$, respectively. This suggests that the sensitivity values of $\mathrm{ZrN}$ - and HfN-based nanoparticle systems are higher than that of commonly used plasmonic material gold. Another important sensor parameter is the figure of merit (FOM), which is simply the product of quality factor $(\mathrm{QF})$ of resonance and the sensitivity $(S)$, and it is defined as [14]

$\mathrm{FOM}(\mathrm{nm} / \mathrm{RIU})=\mathrm{QF} \times \mathrm{S}$

The calculated FOM (nm/RIU) values are $3.5 \times 10^{3}$, $3.1 \times 10^{3}$, and $2.8 \times 10^{3}$ for Au-, $\mathrm{ZrN}-$, and HfN-based nanomatryoshka, respectively. This suggests that the FOM

Table 3 The optimized plasmonic Fano resonant wavelength for different defected concentration of blood plasma and size of nanomatryoshka with $R_{1}=30 \mathrm{~nm}, R_{2}=50 \mathrm{~nm}$, and $R_{3}=70 \mathrm{~nm}$

\begin{tabular}{lllll}
\hline Sr. no & \multirow{2}{*}{$\begin{array}{l}\text { Blood plasma concen- } \\
\text { tration }(\mathrm{g} / \mathrm{l})\end{array}$} & \multicolumn{3}{l}{ Fano wavelength $(\mathrm{nm})$} \\
\cline { 3 - 5 } & & $\mathrm{Au}$ & $\mathrm{ZrN}$ & $\mathrm{HfN}$ \\
\hline 1 & 0 & 737 & 741 & 703 \\
2 & 10 & 740 & 745 & 705 \\
3 & 20 & 743 & 750 & 711 \\
4 & 30 & 747 & 754 & 715 \\
5 & 40 & 750 & 759 & 720 \\
6 & 50 & 755 & 764 & 726 \\
\hline
\end{tabular}

of $\mathrm{ZrN}$ - and HfN-based nanomatryoshka is comparable with that of Au-based nanomatryoshka. The summary of calculated parameters for $\mathrm{Au}-, \mathrm{ZrN}-$, and $\mathrm{HfN}$-based nanomatryoshka is provided in Table 4 . The assessment of Fano resonance-based sensing characteristics shows the following: (I) Scattering efficiencies of $\mathrm{ZrN}$ - and $\mathrm{HfN}$ based nanomatryoshka are comparable with $\mathrm{Au}$, one of the most widely used conventional plasmonic material.
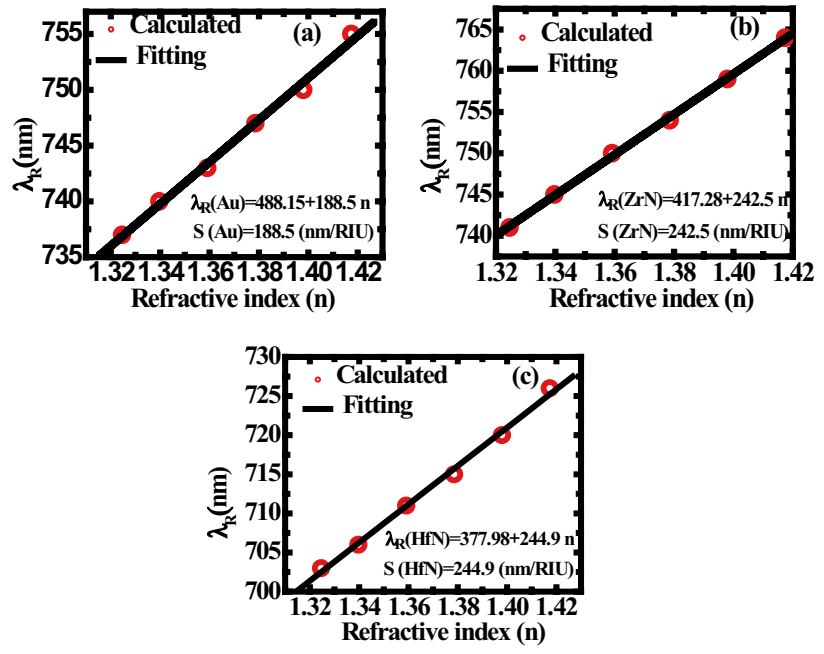

Fig. 6 The calculated dependence of resonant Fano peak position on the concentration and hence the refractive index of the blood plasma medium for $\mathbf{a} \mathrm{Au}, \mathbf{b} \mathrm{ZrN}$, and $\mathbf{c} \mathrm{HfN}$-based nanomatryoshka specified by $R_{1}=30 \mathrm{~nm}, R_{2}=50 \mathrm{~nm}$, and $R_{3}=70 \mathrm{~nm}$ 
Table 4 The optimized plasmonic Fano resonance based sensing parameters for nanomatryoshka with $R_{1}=30 \mathrm{~nm}, R_{2}=50 \mathrm{~nm}$, and $R_{3}=70 \mathrm{~nm}$

\begin{tabular}{lllll}
\hline \multicolumn{4}{l}{ Nanomatryoshka (NMS), $R_{1}=30 \mathrm{~nm}, R_{2}=50 \mathrm{~nm}$, and $R_{3}=70 \mathrm{~nm}$} \\
\hline S. no & Parameters & $\mathrm{Au}$ & $\mathrm{ZrN}$ & $\mathrm{HfN}$ \\
\hline 1 & $\lambda_{R}(\mathrm{~nm})$ & 738 & 743 & 705 \\
2 & $\mathrm{~S}(\mathrm{~nm} / \mathrm{RIU})$ & 188.5 & 242.5 & 244.9 \\
3 & FWHM (nm) & 45.57 & 57.16 & 61.23 \\
4 & FOM (nm/RIU) & $3.5 \times 10^{3}$ & $3.1 \times 10^{3}$ & $2.8 \times 10^{3}$ \\
\hline
\end{tabular}

(II) Refractory transition metal nitrides like $\mathrm{ZrN}$ and $\mathrm{HfN}$ are potential plasmonic materials for Vis-NIR spectral region. (III) Scattering efficiencies of nitride-based nanomatryoshka geometry are extremely sensitive to the thicknesses, aspect ratio, and the refractive index of constituent mediums.

\section{Summary}

In this work, the sensing characteristics of Fano resonancebased plasmonic nanomatryoshka comprising of $\mathrm{Au}, \mathrm{ZrN}$, and HfN plasmonic materials are assessed. It is shown that $\mathrm{Au}, \mathrm{ZrN}$, and HfN nanomatryoshka-based Fano sensors can exhibit the sensitivity $(\mathrm{nm} / \mathrm{RIU}) \sim 188.5,242.5$, and 244.9, respectively. Moreover, the corresponding figure of merit $(\mathrm{nm} / \mathrm{RIU}) \sim 3.5 \times 10^{3}, 3.1 \times 10^{3}$, and $2.8 \times 10^{3} \mathrm{HfN}$ is exhibited. Therefore, biosensors with high sensitivity and figure of merit can be developed by employing Fano modes in plasmonic nanomatryoshka. The availability of compatible nitride-based plasmonic materials can open the door for additional applications. The present research can play a vital role in developing refractory transition metal nitride-based plasmonic systems like solar cells, molecular electronic devices, optical switches, and biosensors.

Authors Contributions Both authors contributed to the study conception and design. The calculations were performed by Pankaj Pathania. Material preparation, manuscript writing, and analysis were performed by Pankaj Pathania and Manmohan Singh Shishodia. Both authors read and approved the final manuscript.

Funding Partial financial support was received from SERB-DST through Grant No. CRG/2019/006912.

Data Availability The data that support the findings of this study are available from the corresponding author upon reasonable request.

\section{Compliance with Ethical Standards}

Competing Interest The authors declare that they have no conflict of interest.

\section{References}

1. Vaupel JW (2010) Biodemography of human ageing. Nature 464:536

2. Angelopoulos A, Bourbakis NG (2010) Prognosis-a wearable health-monitoring system for people at risk: Methodology and modeling. IEEE Trans Inf Technol Biomed 14:613

3. Connerade JP, Lane AM (1988) Interacting resonances in atomic spectroscopy. Rep Prog Phys 51:1439

4. Maier SA, Brongersma ML, Kik PG, Meltzer S, Requicha AAG, Atwater HA et al (2001) Plasmonics—a route to nanoscale optical devices. Adv Mater 13:1501

5. Loiseau A, Asila V, Aullen GB, Lam M, Salmain M, Boujday S et al (2019) Silver-based plasmonic nanoparticles for and their use in biosensing. Biosensors 9:78

6. Singh A, Shishodia MS (2020) Graphene vs. silica coated refractory nitrides based core-shell nanoparticles for nanoplasmonic sensing. Physica E: Low-dimensional Systems and Nanostructures 114288

7. Pathania P, Shishodia MS (2019) Gain assisted transition metal ternary nitride () core-shell based sensing of waterborne bacteria in drinking water. Plasmonics 14:1435

8. Shishodia MS, Pathania P (2018) Estimation of sensing characteristics for refractory nitrides based gain assisted core-shell plasmonic nanoparticles. Phys Plasmas 25:042101

9. Juneja S, Shishodia MS (2018) Surface plasmon amplification in refractory transition metal nitride based nanoparticle dimmers. Opt Commun 433:89

10. Shishodia MS, Perera AGU (2011) Hetero junction plasmonic midinfrared detectors. J Appl Phys 109:043108

11. Shishodia MS, Jayaweera PVV, Matsik SG, Perera AGU, Liu HC, Buchanan M et al (2011) Surface plasmon enhanced IR absorption: Design and experiment. Photonics and Nanostructures: Fundam Appl 9:95

12. Li G, Shishodia MS, Fainberg BD, Apter B, Oren M, Nitzan A, Ratner MA et al (2012) Compensation of coulomb blocking and energy transfer in the current voltage characteristic of molecular conduction junctions. Nano Lett 12:2228

13. Shishodia MS, Juneja S (2019) Surface plasmon enhanced electric field versus Forster resonance energy transfer near core-shell nanoparticle. J Appl Phys 125:213104

14. Tao Y, Guo Z, Zhang A, Zhang J, Wang B, Qu S et al (2015) Gold nanoshells with gain-assisted silica core for ultra-sensitive biomolecular sensors. Opt Comm 349:193

15. Zhou N, Puente VL, Wang Q, Polavarapu L, Santos IP, Xu QH et al (2015) Plasmon-enhanced light harvesting: Applications in enhanced photocatalysis, photodynamic therapy and photovoltaics. RSC Adv 5:29076

16. Orozcoet CA et al (2014) Au nanomatryoshkas as efficient nearinfrared photothermal transducers for cancer treatment: Benchmarking against nanoshells. ACS Nano 8:6372

17. Luk'yanchuk B, Maier S (2010) The Fano resonance in plasmonic nanostructures and metamaterials. Nat. Mater. 9:707

18. Zhang J, Zayats A (2013) Multiple Fano resonances in single-layer nonconcentric core-shell nanostructures. Opt Express 21:8426

19. Wu D, Jiangand S, Liu X (2011) Tunable Fano resonances in three layered bimetallic Au and Ag nanoshell. J Phys Chem C 115:23797

20. Liaw JW, Chen HC, Kuo MK et al (2013) Plasmonic Fano resonance and dip of $\mathrm{Au}-\mathrm{SiO}_{2}-\mathrm{Au}$ nanomatryoshka. Nanoscale Res Lett 8:468

21. Shu J, Gao W, Xu Q et al (2013) Fano resonance in concentric ring apertures. Opt Express 21:11101

22. Mirin NA, Bao K, Nordlander P et al (2009) Fano resonances in plasmonic nanoparticle aggregates. J Phys Chem A 113:4028 
23. Hopkins B, Filonov DS, Glybovski SB, Miroshnichenko AE et al (2015) Hybridization and the origin of Fano resonances in symmetric nanoparticle trimers. Phys Rev B 92:045433

24. Zheng C, Jia T, Zhao H, Zhang S, Feng D, Sun Z et al (2016) Low threshold tunable spaser based on multipolar Fano resonances in disk-ring plasmonic nanostructures. J Phys D: Applied Physics 49:015101

25. Hao F, Nordlander P, Sonnefraud Y, Van Dorpe P, Maier SA et al (2009) Tunability of subradiant dipolar and Fano-type plasmon resonances in metallic ring/disk cavities: Implications for nanoscale optical sensing. ACS Nano 3:643

26. Ye J, Wen F, Sobhani H, Lassiter JB, Dorpe PV, Nordlander P, Halas NJ et al (2012) Plasmonic nanoclusters: Near field properties of the Fano resonance interrogated with sers. Nano Lett $12: 1660$

27. Miroshnichenko AE, Flach S, Kivshar YS et al (2010) Fano resonances in nanoscale structures. Rev Mod Phys 82:2257

28. Chang WS, Lassiter JB, Swanglap P, Sobhani H, Khatua S, Nordlander P, Halas NJ, Link S (2012) A plasmonic fano switch . Nano Lett 12(9):4977

29. Zeng B, Gao Y, Bartoli FJ et al (2014) Rapid and highly sensitive detection using Fano resonances in ultrathin Plasmonic nanogratings. Appl Phys Lett 105:161106

30. He J, Fan C, Wang J, Ding P, Cai G, Cheng Y, Zhu S, Liang E et al (2013) A giant localized field enhancement and high sensitivity in an asymmetric ring by exhibiting Fano resonance. J Opt 15:025007

31. Yu Y, Xue W, Semenova E, Yvind K, Mork J et al (2017) Demonstration of a self-pulsing photonic crystal fano laser. Nat Photon 11:81

32. Wu C et al (2012) Fano-resonant asymmetrical metamaterials for ultrasensitive spectroscopy and identification of molecular monolayers. Nat Mater 11:69

33. Heeg KP, Ott C, Schumacher D, Even J et al (2015) Interferometric phase detection at X-ray energies via Fano resonance control. Phys Rev Lett 114:207401

34. Fang C, Lee YH, Shao L, Jiang R, Wang J, Qing-Hua Xu (2013) Correlating the plasmonic and structural evolutions during the sulfidation of Ag nanocubes. ACS Nano 7:9354

35. Cortie MB, Arnold MD, Keast VJ (2019) The quest for zero loss: Unconventional materials for plasmonics. Adv Mater 32:1904532

36. Naik GV, Shalaev VM, Boltasseva A et al (2013) Alternative plasmonic materials: Beyond gold and silver. Adv Mater 25:3264

37. Guler U, Shalaev VM, Boltasseva A et al (2015) Nanoparticle plasmonics: Going practical with transition metal nitrides. Mater Today 18:227

38. Xian Y, Cai Y, Sun X, Liu X, Guo Q, Zhang Z, Tong L, Qiu J et al (2019) Refractory plasmonic metal nitride nanoparticles for broadband near-infrared optical switches. Laser Photonics Rev 13:1900029

39. Kumar M, Ishii S, Umezawa N, Nagao T et al (2016) Band engineering of ternary metal nitride system $\mathrm{Ti}_{1-\mathrm{x}} \mathrm{Zr}_{\mathrm{x}} \mathrm{N}$ for plasmonic applications. Opt Mat Express 6:1
40. Guler U, Naik GV, Boltasseva A, Shalaev VM, Kildishev AV et al (2012) Performance analysis of nitride alternative plasmonic materials for localized surface plasmon applications. Appl Phys B 012:4955

41. Liu PY, Chin LK, Ser W, Chen HF, Hsieh M, Lee H et al (2016) Cell refractive index for cell biology and disease diagnosis: Past, present and future. Lab on Chip 16:634

42. Liu PY, Chin LK, Serand BW, Ayi TC et al (2014) An optofluidic imaging system to measure the biophysical signature of single waterborne bacteria. Lab on Chip 14:4237

43. Coles HJ, Jennings BR, Morris VJ et al (1975) Refractive index increment measurement for bacterial suspensions. Phys Med Biol 20:310

44. Karunya R, Jaypraksh KS, Senet AK (2019) Rapid measurement of hydrogen sulphide in human blood plasma using microfludic method. Sci reports 9:3258

45. Roekmono H, Hadiand HN (2018) Imtihani, Glucose and cholesterol in blood plasma are sensed by $\mathrm{ZnO}$ based paper based microfludics techniques. Int Drug delivery tech 8:153

46. Pan ZB (2018) A silver metal complex as a luminescent probe for enzymatic sensing of glucose in blood plasma and urine. Dalton Trans 47:8346

47. Garbett NC, Merchant ML, William Helm C (2014) Detection of cervical cancer biomarker patterns in blood plasma and urine by deferential scanning calorimeter and mass spectrometry. PLoS ONE9

48. Kim J, Kim S, Nguyen TT, Lee R et al (2016) Label free quantitative immunoassay of fibrinogenin alzheimer's diseases patients plasma using fiber optical surface Plasmon resonance. J Electron Mater 45:2354

49. Adenand AL, Kerker M (1951) Scattering of electromagnetic waves from two concentric spheres. J Appl Phys 22:601

50. Wu D, Xu X, Liu X et al (2008) Tunable near-infrared optical properties of three-layered metal nanoshells. J Chem Sci 129:074711

51. Wu ZS, Wang YP (1991) Electromagnetic scattering for multilayered sphere: Recursive algorithms. Radio Science 26:1393

52. Wang M, Cao M, Chen X, Gu N et al (2011) Subradiant plasmon modes in multilayer metal-dielectric nanoshells. J Phys Chem C 115:20920

53. Zhang Y, Jia T, Zhang $\mathrm{H}, \mathrm{Xu} \mathrm{Z}$ et al (2012) Fano resonances in disk-ring plasmonic nanostructure: Strong interaction between bright dipolar and dark multipolar mode. Opt Lett 37:4919

54. Qin Xie G, Xi Dong BX, Wang and W.Q. Huang, (2018) High-Q Fano resonance in terahertz frequency based on an asymmetric metamaterial resonator. Nanoscale Res Lett 13:294

55. Hala J, Khozondar E, Mahalakshmi P, Amiriand IS, Yupapin P (2019) Design of one dimensional refractive index sensor using ternary photonic crystal waveguide for plasma blood samples applications. Physica E 111:29

Publisher's Note Springer Nature remains neutral with regard to jurisdictional claims in published maps and institutional affiliations. 